\title{
Novel Wavelet-Based Segmentation of Prostate CBCT Images with Implanted Calypso Transponders
}

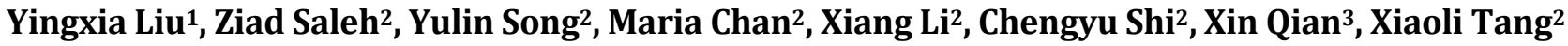 \\ ${ }^{1}$ Shandong Communication and Media College, Jinan, China \\ ${ }^{2}$ Medical Physics Department, Memorial Sloan Kettering Cancer Center, New York, NY, USA \\ ${ }^{3}$ Radiation Oncology, North Shore Long Island Jewish Health System, New Hyde Park, NY, USA \\ Email: tangx@mskcc.org
}

How to cite this paper: Liu, Y.X., Saleh, Z., Song, Y.L., Chan, M., Li, X., Shi, C.Y., Qian, X. and Tang, X.L. (2017) Novel Wavelet-Based Segmentation of Prostate CBCT Images with Implanted Calypso Transponders. International Journal of Medical Physics, Clinical Engineering and Radiation Oncology, 6, 336-343.

https://doi.org/10.4236/ijmpcero.2017.63030

Received: July 7, 2017

Accepted: August 27, 2017

Published: August 30, 2017

Copyright () 2017 by authors and Scientific Research Publishing Inc. This work is licensed under the Creative Commons Attribution International License (CC BY 4.0).

http://creativecommons.org/licenses/by/4.0/

(c) (7) Open Access

\begin{abstract}
Segmentation of prostate Cone Beam CT (CBCT) images is an essential step towards real-time adaptive radiotherapy (ART). It is challenging for Calypso patients, as more artifacts generated by the beacon transponders are present on the images. We herein propose a novel wavelet-based segmentation algorithm for rectum, bladder, and prostate of $\mathrm{CBCT}$ images with implanted $\mathrm{Ca}$ lypso transponders. For a given CBCT, a Moving Window-Based Double Haar (MWDH) transformation is applied first to obtain the wavelet coefficients. Based on a user defined point in the object of interest, a cluster algorithm based adaptive thresholding is applied to the low frequency components of the wavelet coefficients, and a Lee filter theory based adaptive thresholding is applied on the high frequency components. For the next step, the wavelet reconstruction is applied to the thresholded wavelet coefficients. A binary (segmented) image of the object of interest is therefore obtained. 5 hypofractionated Calypso prostate patients with daily CBCT were studied. DICE, Sensitivity, Inclusiveness and $\Delta \mathrm{V}$ were used to evaluate the segmentation result.
\end{abstract}

\section{Keywords}

CBCT, Prostate Segmentation, Wavelets, MWDH

\section{Introduction}

One important step of the Adaptive Radiation Therapy (ART) is the segmentation of the $\mathrm{CBCT}$ images-a step required for the adaptive planning. This is specially important for hypofractionated treatments. Many studies have been published on automatic segmentation of prostate CBCT [1]-[9]. Accurate segmenta- 
tion of CBCT is challenging due to the daily variations in rectal and bladder fillings as well as the increased noise levels in CBCT images. In our institution, some hypofractionated prostate patients are treated by Calypso tracking system (Varian, Palo Alto, CA). Three beacon transponders are implanted to the prostate, so that the target motion during the treatment delivery can be tracked in real time. However, the metal transponders introduce artifacts to the $\mathrm{CBCT}$ imaging, which makes segmentation more challenging. Based on our knowledge, no study was published yet on the segmentation of prostate CBCT with implanted Calypso transponders.

We propose to segment prostate and surrounding structures in the wavelet domain. The major advantage of wavelets is the ability to perform local analysis, i.e. trends, breakdown points, discontinuities, etc. The Double Haar wavelet transform can make the image edge detection more effective. The moving window implementation can protect the details and smooth the noise [10]. Therefore, we use a combination of these two-the Moving window-based Double Haar (MWDH) transformation for our prostate segmentation.

Adapted thresholds are assigned to different frequency components after MWDH. In low frequency component, cluster algorithm is employed to obtain a threshold $T_{L}$ to classify the region of interest. In high frequency components, Lee filter theory is used to calculate the adaptive threshold $T_{H}$ after de-noising. The segmented result is obtained by wavelet reconstruction of the thresholded components. Physician contoured the structures, and these served as ground truth.

The rest of this paper is organized as follows: in section 2, we will present the wavelet based segmentation algorithm in more details. The experimental results are shown in section 3 and followed by discussion. The last section will conclude this study.

\section{Materials and Methods}

The flow chart of the proposed algorithm is shown in Figure 1.

\subsection{Moving Window Based Double Haar Wavelet Transform}

Different than the conventional two-channel wavelet transform, the Double Haar wavelet transform (DHWT) has three channels. As shown in Figure 2, the input signal $x(n)$ is filtered by one low pass filter $H_{0}(z)$ and two high pass filters $H_{1}(z)$ and $H_{2}(z), x_{0}(n)$ (low frequency component/sub-band) and $x_{1}(n)$ and $x_{2}(n)$ (high frequency components/sub-bands) are the outputs. Then, sub-sampling is applied on each component to keep image size the same. For reconstruction, the interpolation needs to be applied first, followed by the reconstruction filters $G_{0}(z), G_{1}(z)$, and $G_{2}(z)$.

\subsection{The Adaptive Thresholding}

In wavelet domain, low frequency components usually represent the main characteristics or identity of an image. The high frequency components, on another 


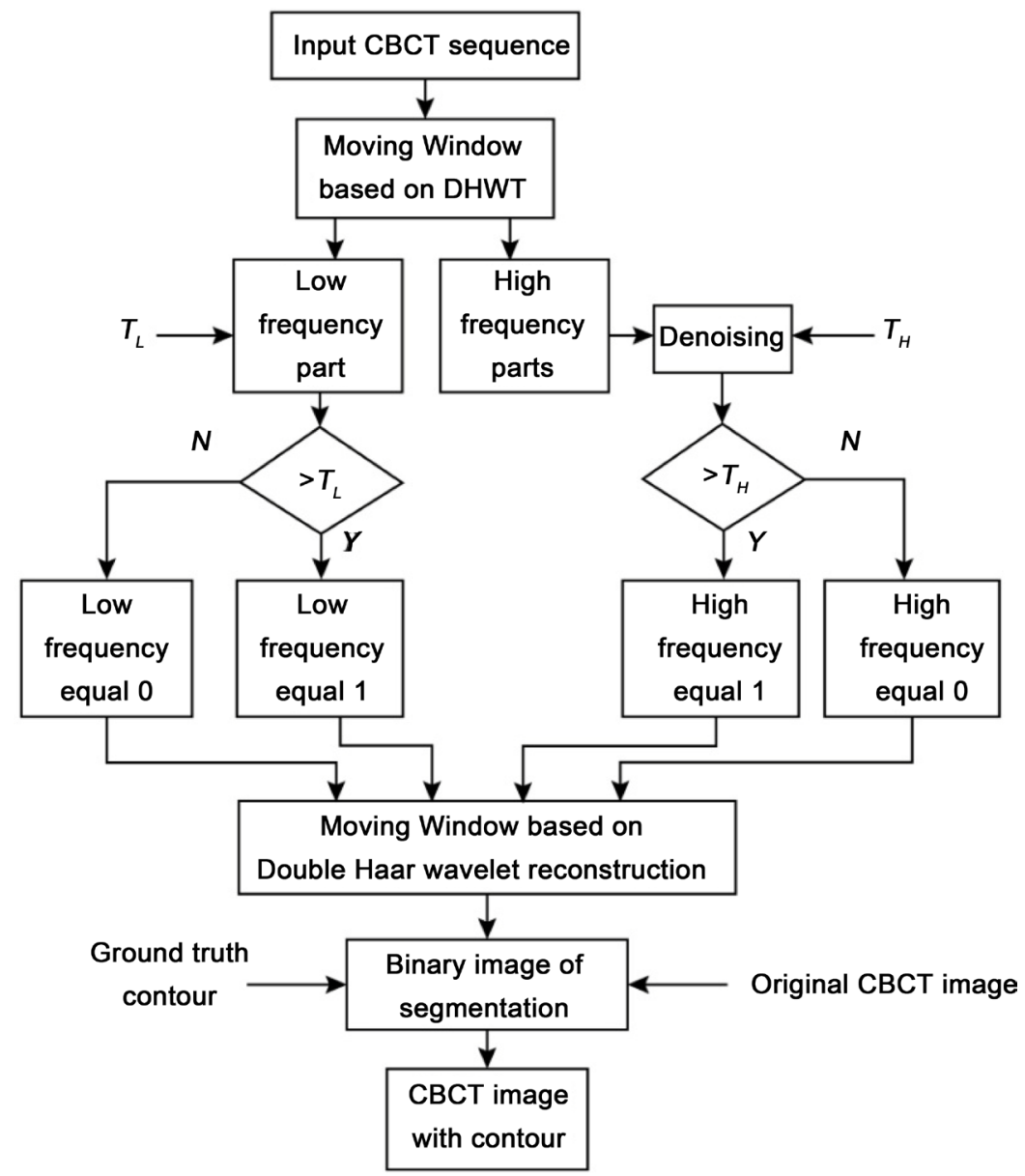

Figure 1. The flow chart of the segmentation algorithm based on MWDH. $T_{L}$ and $T_{H}$ are the thresholds for low and high frequency components.

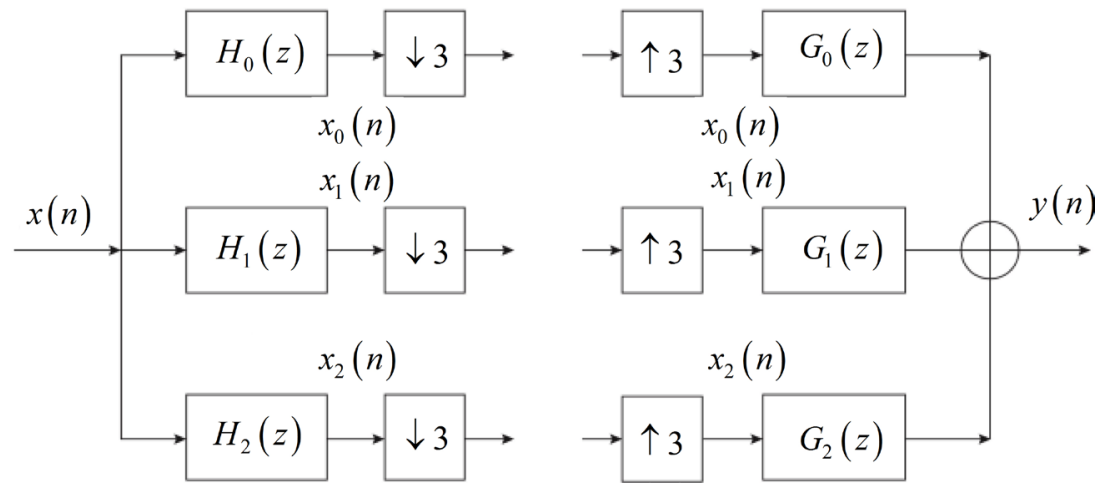

Figure 2. The structure of DHWT.

hand, are the nuance or details of an image. Considering these differences, different thresholding methods were applied on high and low components.

For low frequency component, the goal of thresholding is to group pixels of similar properties to a same group. Cluster algorithm [11] was designed to achieve this goal and was applied for low frequency component thresholding.

LEE filter can yield a local linear minimum mean-square error estimate of the original and give a better edge protective effect. Therefore, it was utilized for 
high frequency components thresholding.

For both cluster algorithm and LEE filter, image pixel statistics is needed to calculate the thresholding value. This is achieved by having user manually select a point in the structure of interest before the segmentation. Then a window is applied to collect the statistics.

\subsection{Patient Information}

Five hypofractionated prostate patients with prescription of $800 \mathrm{cGy} \times 3$ and daily CBCT were studied. Each patient had 3 Calypso transponder beacons implanted, and the patients were setup and treated with Calypso tracking system. Two sets of CBCT image from each patient were studied. $3 \times 3$ moving window was used. The MWDH based segmentation algorithm was applied to segment and prostate, bladder and rectum.

\subsection{Evaluation of the Segmentation}

The structures were also contoured by trained expert, and these served as ground truth. We validate the proposed segmentation algorithm using the following metrics.

Dice Similarity Coefficient (DSC): DSC measures the spatial overlap between two segmentations [12], and is defined as:

$$
D S C=\frac{2\left(V_{\text {seg }} \cap V_{\text {ground }}\right)}{V_{\text {seg }}+V_{\text {ground }}}
$$

where $V_{\text {seg }}$ is the structure volume obtained by the proposed segmentation algorithm. $V_{\text {ground }}$ is the ground truth. DSC has a range of $[0,1]$, where 0 means no overlap, and 1 means complete overlap.

Sensitivity: The sensitivity reflects the probability that the automatic segmentation contour match the ground truth contour [12]. It is defined as:

$$
\text { Sensitivity }=\frac{V_{\text {seg }} \cap V_{\text {ground }}}{V_{\text {ground }}}
$$

Inclusiveness (Incl): The inclusiveness shows the inclusion of $V_{\text {seg }}$ within $V_{\text {ground }}$, it reflects the probability that a pixel of the $V_{\text {seg }}$ also belongs to $V_{\text {ground }}$. It is computed by:

$$
\text { IncI }=\frac{V_{\text {seg }} \cap V_{\text {ground }}}{V_{\text {seg }}}
$$

$\Delta V \vdots \Delta V$ is the ratio of the difference between $V_{\text {seg }}$ and $V_{\text {ground }}$ over $V_{\text {ground }}$, it is defined as:

$$
\Delta V=\frac{V_{\text {seg }}-V_{\text {ground }}}{V_{\text {ground }}} \times 100 \%
$$

\section{Results}

Table 1 lists the statistical results of the segmentation. Figure 3 displays examples of the segmentation results. 
Table 1. Statistical results.

\begin{tabular}{ccccccc}
\hline Structure & Metrics & Patient 1 & Patient 2 & Patient 3 & Patient 4 & Patient 5 \\
\hline \multirow{4}{*}{ Rectum } & DICE & 0.891 & 0.901 & 0.838 & 0.877 & 0.709 \\
& Sensitivity & 0.954 & 0.899 & 0.98 & 0.976 & 0.758 \\
& Inclusiveness & 0.836 & 0.846 & 0.734 & 0.797 & 0.983 \\
& $\Delta \mathrm{V}$ & $15.53 \%$ & $12.17 \%$ & $34.30 \%$ & $22.80 \%$ & $27.27 \%$ \\
\multirow{5}{*}{ Prostate } & DICE & 0.773 & 0.818 & 0.811 & 0.87 & 0.888 \\
& Sensitivity & 0.926 & 0.886 & 0.933 & 0.928 & 0.938 \\
& Inclusiveness & 0.677 & 0.807 & 0.62 & 0.834 & 0.817 \\
& $\Delta \mathrm{V}$ & $46.47 \%$ & $36.90 \%$ & $36.40 \%$ & $23.30 \%$ & $13.40 \%$ \\
& DICE & 0.86 & 0.811 & 0.926 & 0.919 & 0.927 \\
& Sensitivity & 0.832 & 0.765 & 0.953 & 0.912 & 0.939 \\
& Inclusiveness & 0.912 & 0.92 & 0.899 & 0.931 & 0.919 \\
& $\Delta \mathrm{V}$ & $14.73 \%$ & $20.63 \%$ & $10.13 \%$ & $7.40 \%$ & $2.95 \%$ \\
\hline
\end{tabular}
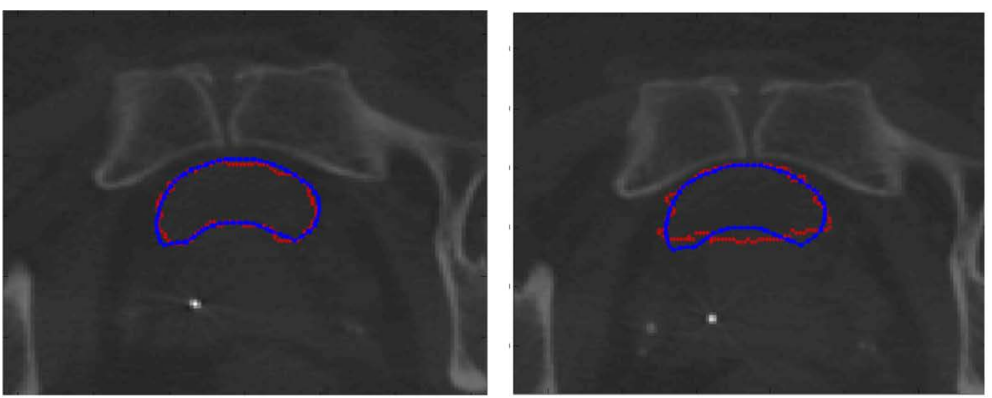

(a)
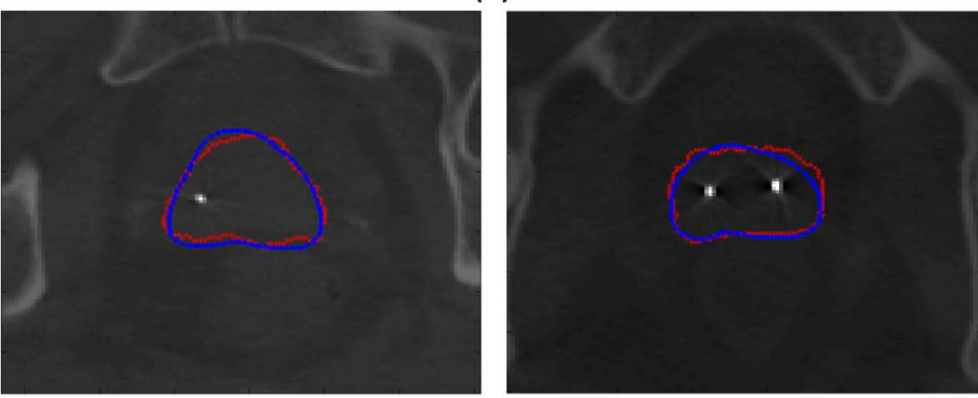

(b)
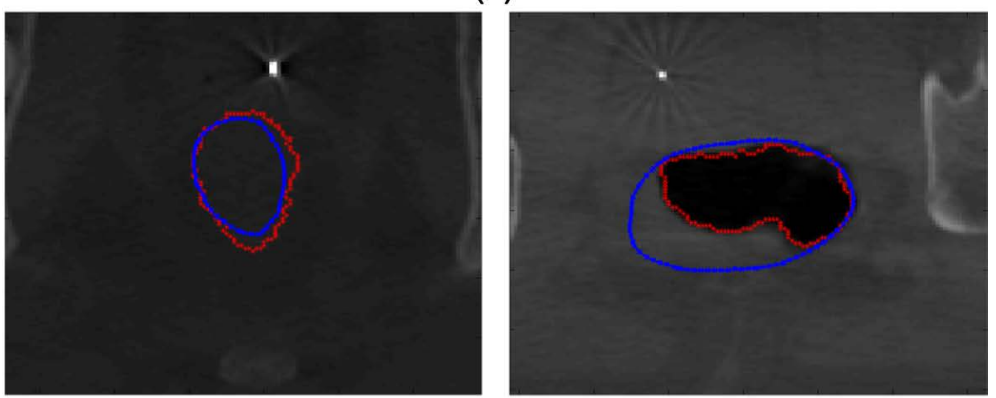

(c)

Figure 3. Contour comparison between the segmentation (red) and ground truth (blue). The ones with inferior segmentation results were plotted using thicker lines. (a) Bladder; (b) Prostate; (c) Rectum. 


\section{Discussion}

Segmentation of prostate in important for adaptive radiation therapy [13]-[20]. One type of commonly used approach is deformable registration-based algorithm [4] [9]. Usually, a rigid registration is applied first to propagate the planning CT contours to the CBCT images. Then, iterative algorithm (i.e. Demons) is applied to calculate the pixel deformation flow/vector, until the given constrain is met. This works for some cases. However, when a large fraction of the propagated rectum and bladder contours were unacceptable, it provided a sub-optimal starting point for the deformable registration. This approach is difficult to generalize-the same measures and transformation constraints might not work for all the structures.

Another type is model/atlas based segmentation. As its name suggests, model needs to be built. The assumption of this approach is that structures of interest have a repetitive form of geometry. It involves expert manual segmentation of the structures of interest, and registration of the training examples to a common pose or model training to build the model [20] [21] [22]. This type involves significant amount of expert time. Expert contours usually involve human variations as well, which might be reflected in the built segmentation model.

Our proposed wavelet based prostate CBCT segmentation algorithm does not require deformable registration or model building. The moving window-based MWDH transfers the СBCT images to the wavelet domain, which contains high and low frequency components. We applied different thresholding technique to segment the different components. The final segmentation was achieved by the reconstruction of the thresholded wavelet coefficients. The algorithm is semi-automatic, as it requires user input to select a starting point. Our proposed algorithm achieved reasonable DICE index for all structures over all patients. However, it has challenges in two scenarios: 1) prostate with very low contrast; 2) rectum with significant amount of gas. The right hand side of Figure $3(\mathrm{~b})$ illustrates the first scenario. The contrast of prostate was low, and the algorithm over-segmented the prostate. The right hand side of Figure 3(c) illustrates the second scenario, when the rectum was filled with significant amount of gas, the algorithm tended to segment the gas. We have tried to select the user point inside and outside of the gas, and did not observe noticeable improvement. Multiple user entered points might help these two kinds of situations, and we will include it in our future work.

Haar wavelet transform has many advantages. There is no need for multiplications. It requires only additions and therefore the computation time is short. Its input and output length are the same. Although the double Haar wavelet transform enhanced its ability to analyze the localized features of images, other more sophisticated wavelet transforms might analyze the high frequency components better and further improve the segmentation results.

\section{Conclusion}

The proposed algorithm appeared effective segmenting prostate CBCT images 
with the present of the Calypso artifacts under most common clinical scenarios. However, when the prostate contrast is low or there is significant amount of gas in the rectum, the algorithm might have inferior segmentation result.

\section{References}

[1] Kim, J., Kumar, S., Liu, C., Zhong, H., Pradhan, D., Shah, M., Cattaneo, R., Yechieli, R., Robbins, J.R., Elshaikh, M.A. and Chetty, I.J. (2013) A Novel Approach for Establishing Benchmark CBCT/CT Deformable Image Registrations in Prostatecancer Radiotherapy. Physics in Medicine and Biology, 58, 8077-8097. https://doi.org/10.1088/0031-9155/58/22/8077

[2] Kim, J., Hammoud, R., Pradhan, D., Zhong, H., Jin, R.Y., Movsas, B. and Chetty, I.J. (2010) Prostate Localization on Daily Cone-Beam Computed Tomography Images: Accuracy Assessment of Similarity Metrics. Radiation Oncology Biology Physics, 77, 1257-1265. https://doi.org/10.1016/j.ijrobp.2009.09.068

[3] Deegan, T., Owen, R., Holt, T., Fielding, A., Biggs, J., Parfitt, M., Coates, A. and Roberts, L. (2015) Assessment of Cone Beam CT Registration for Prostate Radiation Therapy: Fiducial Marker and Soft Tissue Methods. Journal of Medical Imaging and Radiation Oncology, 59, 91-98. https://doi.org/10.1111/1754-9485.12197

[4] Thor, M., Petersen, J.B., Bentzen, L., Hoyer, M. and Muren, L.P. (2011) Deformable Image Registration for Contour Propagation from CT to Cone-Beam CT Scans in Radiotherapy of Prostate Cancer. Acta Oncologic, 50, 918-925.

[5] Paluskaa, P., Hanusa, J., Sefrovab, J., Rouskovab, L., Grep, J., Jansab, J., Kasaovab, L., Hodekb, M., Zouharb, M., Vosmikb, M. and Peterab, J. (2012) Utilization of Cone-Beam CT for Offline Evaluation of Target Volume Coverage during Prostate Image-Guided Radiotherapy Based on Bony Anatomy Alignment. Reports of Practical Oncology and Radiotherapy, 17, 134-140.

[6] Warfield, S.K., Zou, K.H., Kaus, M.R. and Wells, W.M. (2002) Simultaneous Validation of Image Segmentation and Assessment of Expert Quality. International Symposium on Biomedical Imaging, 7, 7-10. https://doi.org/10.1109/ISBI.2002.1029201

[7] Warfield, S.K., Zou, K.H. and Wells, W.M. (2002) Validation of Image Segmentation and Expert Quality with Anization Algorithm. Proceedings of 5 th International Conference on Medical Imaging Computing and Computer Assisted Interventions, 9, 22-25.

[8] Young, A.V., Wortham, A., Wernick, I., Evans, A. and Ennis, R.D. (2011) Atlas-Based Segmentation Improves Consistency and Decreases Time Required for Contouring Postoperative Endometrial Cancer Nodal. Radiation Oncology Biology Physics, 79, 943-947. https://doi.org/10.1016/j.ijrobp.2010.04.063

[9] Lawson, J.D., Schreibmann, E., Jani, A.B. and Fox, T. (2007) Quantitative Evaluation of a Cone-Beam Computed Tomography Planning Computed Tomography Deformable Image Registration Method for Adaptive Radiation Therapy. Journal of Applied Clinical Medical Physics, 8, 2432. https://doi.org/10.1120/jacmp.v8i4.2432

[10] Venkateswarlu, G., JayanthNadh, B. and Hymavathi, B. (2012) Image Enhancement Using Moving Window-Based Double Haar Wavelet Transform. International Journal of Emerging Technology and Advanced Engineering, Vol. 2.

[11] Zhang, T. (2006) Automatic Target Recognition. Hubei Science and Technology Publishing Press.

[12] Macchia, M.L., Fellin, F., Amichetti, M., Cianchetti, M., Gianolini, S., Paola, V., 
Lomax, A.J. and Widesott, L. (2012) Systematic Evaluation of Three Different Commercial Software Solutions for Automatic Segmentation for Adaptive Therapy in Head-and-Neck, Prostate and Pleural Cancer. Radiation Oncology, 7, 160-176. https://doi.org/10.1186/1748-717X-7-160

[13] Hwee, J., Louie, A.V., Gaede, S., Bauman, G., D’Souza, W., Sexton, T., Lock, M., Ahmad, B. and Rodrigues, G. (2011) Technology Assessment of Automated Atlas Based Segmentation in Prostate Bed Contouring. Radiation Oncology Biology Physics, 6, 110.

[14] Zelefsky, M.J., Kollmeier, M. and Cox, B. (2012) Improved Clinical Outcomes with High-Dose Image Guided Radiotherapy Compared with Non-IGRT for the Treatment of Clinically Localized Prostate Cancer. Radiation Oncology Biology Physics, 84, 125-129.

[15] Gill, S., Li, J. and Thomas, J. (2012) Patient-Reported Complications from Fiducial Marker Implantation for Prostate Image-Guided Radiotherapy. Radiation Oncology Biology Physics, 85, 1011-1017.

[16] Kok, D., Gill, S., Bressel, M., Kron, T., Fox, C., Duchesne, G., Tai, K.H. and Foroudi, F. (2013) Late Toxicity and Biochemical Control in 554 Prostate Cancer Patients Treated with and without Dose Escalated Image Guided Radiotherapy. Radiation Oncology Biology Physics, 107, 140-146.

[17] Deegan, T., Owen, R., Holt, T., Roberts, L., Biggs, J., McCarthy, A., Parfitt, M. and Fielding, A. (2013) Inter-Observer Variability of Radiation Therapists Aligning to Fiducial Markers for Prostate Radiation Therapy. Medicine Imaging Radiation Oncology, 57, 519-523. https://doi.org/10.1111/1754-9485.12055

[18] Stephen, J., Gardner, J., Wen, N., Kim, J., Liu, C., Pradhan, D., Aref, I., Cattaneo, R., Vance, S., Movsas, B., Chetty, I.J. and Elshaikh, M.A. (2015) Contouring Variability of Human- and Deformable-Generated Contours in Radiotherapy for Prostate Cancer. Physics Medicine Biology, 60, 4429-4447. https://doi.org/10.1088/0031-9155/60/11/4429

[19] Wang, X. (2006) Moving Window-Based Double Haar Wavelet Transform for Image Processing. IEEE Transactions on Image Processing, 15, 2771-2779. https://doi.org/10.1109/TIP.2006.877316

[20] Shi, Y., Liao, S., Zhang, D., Gao, Y. and Shen, D. (2013) Prostate Segmentation in CT Images via Spatial-Constrained Transudative Lasso. Proceedings. IEEE Computer Society Conference on Computer Vision and Pattern Recognition Journal, 10, 1109.

[21] Shi, Y., Liao, S., Zhang, D., Gao, Y. and Shen, D. (2015) Semi-Automatic Segmentation of Prostate in CT Images via Coupled Feature Representation and Spatial-Constrained Transudative Lasso. The IEEE Transactions on Pattern Analysis and Machine Intelligence, 37, 2286-2303. https://doi.org/10.1109/TPAMI.2015.2424869

[22] Kiaei, A. and Khotanlou, H. (2017) Segmentation of Medical Images Using Mean Value Guided Contour. Medical Image Analysis, 40, 111-132. 
Submit or recommend next manuscript to SCIRP and we will provide best service for you:

Accepting pre-submission inquiries through Email, Facebook, LinkedIn, Twitter, etc. A wide selection of journals (inclusive of 9 subjects, more than 200 journals)

Providing 24-hour high-quality service

User-friendly online submission system

Fair and swift peer-review system

Efficient typesetting and proofreading procedure

Display of the result of downloads and visits, as well as the number of cited articles Maximum dissemination of your research work

Submit your manuscript at: http://papersubmission.scirp.org/

Or contact ijmpcero@scirp.org 